\title{
Quantization of Electromagnetic Field and Potential in Rindler Space-Time
}

\author{
Sangwha-Yi* \\ Department of Math, Taejon University 300-716, South korea.
}

*Corresponding Author: Sangwha-Yi, Department of Math, Taejon University 300-716, South korea.

\begin{abstract}
The article treats quantization of electromagnetic field that is defined in Rindler space-time. Likely the electromagnetic field, the potential did quantizated in inertial frame, the electromagnetic field, the potential can quantizate by the transformation of electromagnetic field or the transformation of the potential in the accelerated frame. We treat Lorentz gauge condition in quantization of electromagnetic potential.
\end{abstract}

Keywords: Rindler space-time, Potential quantization, Field quantization, Lorentz Gauge condition

PACS Number: 04.90.+e,03.65

\section{INTRODUCTION}

The article treats quantization of electromagnetic field that is defined in Rindler space-time.

Rindler coordinate transformations are

$$
\begin{aligned}
& c t=\left(\frac{c^{2}}{a_{0}}+\xi^{1}\right) \sinh \left(\frac{a_{0}}{c} \xi^{0}\right), x=\left(\frac{c^{2}}{a_{0}}+\xi^{1}\right) \cosh \left(\frac{a_{0} \xi^{0}}{c}\right)-\frac{c^{2}}{a_{0}} \\
& y=\xi^{2}, z=\xi^{3}
\end{aligned}
$$

At first, in inertial frame, the quantization of electromagnetic potential is [2]

$$
A_{\mu}(x)=\int \frac{d^{3} k}{(2 \pi)^{3} 2 k_{0}} \sum_{\lambda=0}^{3} \varepsilon_{\mu}^{(\lambda)}(k)\left[a^{(\lambda)}(k) \exp (-k \cdot f+i \omega t)+a^{(\lambda)+}(k) \exp (k \cdot f-i \omega t)\right]
$$

In this time, $\quad|q|=\frac{\omega}{c}, k=\left(k_{1}, k_{2}, k_{3}\right), \varepsilon^{(\lambda)} \cdot \varepsilon^{\left(\lambda^{\prime}\right)}=g^{\lambda \lambda^{\prime}}$

$$
\begin{aligned}
& \ddot{k} \cdot \dot{x}-i \omega t=i k x+i k_{2} y+i k_{3} z-i \omega t \\
& =i k\left[\left(\frac{c^{2}}{a_{0}}+\xi^{1}\right) \cosh \left(\frac{a_{0} \xi^{0}}{c}\right)-\frac{c^{2}}{a_{0}}\right]+i k_{2} \xi^{2}+i k_{3} \xi^{3}-i \frac{\omega}{c}\left[\left(\frac{c^{2}}{a_{0}}+\xi^{1}\right) \sinh \left(\frac{a_{0} \xi^{0}}{c}\right)\right]
\end{aligned}
$$

According to [1], the quantization of Electromagnetic potential is in Rindler space-time,

$$
\phi_{\xi}=\frac{1}{\left(1+\frac{a_{0}}{c^{2}} \xi^{1}\right)}\left[\phi \cosh \left(\frac{a_{0} \xi^{0}}{c}\right)-A_{x} \sinh \left(\frac{a_{0} \xi^{0}}{c}\right)\right]
$$




$$
\begin{gathered}
=\int \frac{d^{3} k}{(2 \pi)^{3} 2 k_{0}} \sum_{\lambda=0}^{3} \varepsilon_{\xi^{0}}^{(\lambda)}(k)\left[a^{(\lambda)}(k) \exp (-\dot{k} \cdot f+\dot{\omega} t)+a^{(\lambda)+(k) \exp (k \cdot f-i \omega t)]}\right. \\
\varepsilon_{\xi^{0}}^{(\lambda)}(k)=\frac{1}{\left(1+\frac{a_{0}}{c^{2}} \xi^{1}\right)}\left[\varepsilon_{0}^{(\lambda)}(k) \cosh \left(\frac{a_{0} \xi^{0}}{c}\right)-\varepsilon_{1}^{(\lambda)}(k) \sinh \left(\frac{a_{0} \xi^{0}}{c}\right)\right]
\end{gathered}
$$

Hence, the quantization of Electromagnetic potential in X-axis

$$
\begin{aligned}
& A_{\xi^{1}}=-\phi \sinh \left(\frac{a_{0} \xi^{0}}{c}\right)+A_{x} \cosh \left(\frac{a_{0} \xi^{0}}{c}\right) \\
& =\int \frac{d^{3} k}{(2 \pi)^{3} 2 k_{0}} \sum_{\lambda=0}^{3} \varepsilon_{\xi^{1}}^{(\lambda)}(k)\left[a^{(\lambda)}(k) \exp (-k \cdot f+i \omega t)+a^{(\lambda)+}(k) \exp (k \cdot f-i \omega t)\right] \\
& \varepsilon_{\xi^{1}}^{(\lambda)}(k)=-\varepsilon_{0}^{(\lambda)}(k) \sinh \left(\frac{a_{0} \xi^{0}}{c}\right)+\varepsilon_{1}^{(\lambda)}(k) \cosh \left(\frac{a_{0} \xi^{0}}{c}\right)
\end{aligned}
$$

So, the quantization of Electromagnetic potential in Y-axis

$$
\begin{aligned}
& A_{\xi^{2}}=A_{y} \\
&= \int \frac{d^{3} k}{(2 \pi)^{3} 2 k_{0}} \sum_{\lambda=0}^{3} \varepsilon_{2}^{(\lambda)}(k)\left[a^{(\lambda)}(k) \exp (-k \cdot f+i \omega t)+a^{(\lambda)+}(k) \exp (k \cdot f-i \omega t)\right] \\
&= \int \frac{d^{3} k}{(2 \pi)^{3} 2 k_{0}} \sum_{\lambda=0}^{3} \varepsilon_{\xi^{2}}^{(\lambda)}(k)\left[a^{(\lambda)}(k) \exp (-k \cdot f+i \omega t)+a^{(\lambda)+}(k) \exp (k \cdot f-i \omega t)\right] \\
& \varepsilon_{\xi^{2}}^{(\lambda)}(k)=\varepsilon_{2}^{(\lambda)}(k)
\end{aligned}
$$

Therefore, the quantization of Electromagnetic potential in Z-axis

$$
\begin{aligned}
& A_{\xi^{3}}=A_{z} \\
& =\int \frac{d^{3} k}{(2 \pi)^{3} 2 k_{0}} \sum_{\lambda=0}^{3} \varepsilon_{3}^{(\lambda)}(k)\left[a^{(\lambda)}(k) \exp (-k \cdot f+i \omega t)+a^{(\lambda)+}(k) \exp (k \cdot f-i \omega t)\right] \\
& =\int \frac{d^{3} k}{(2 \pi)^{3} 2 k_{0}} \sum_{\lambda=0}^{3} \varepsilon_{\xi^{3}}^{(\lambda)}(k)\left[a^{(\lambda)}(k) \exp (-k \cdot f+i \omega t)+a^{(\lambda)+}(k) \exp (k \cdot f-i \omega t)\right] \\
& \varepsilon_{\xi^{3}}^{(\lambda)}(k)=\varepsilon_{3}^{(\lambda)}(k)
\end{aligned}
$$

Lorentz gauge condition is in inertial frame and accelerated frame in quantization of electromagnetic potential[1],

$$
\begin{aligned}
& 0=\frac{1}{c} \frac{\partial \phi}{\partial t}+\rho \cdot \rho=\frac{1}{c} \frac{\partial \phi_{\xi}}{\partial \xi^{0}}+\nabla_{\xi} \cdot A_{\xi}+\frac{1}{\left(1+\frac{a_{0} \xi^{1}}{c^{2}}\right)} \frac{a_{0}}{c^{2}} A_{\xi^{1}} \\
& \varepsilon_{0}^{(\lambda)}(k) i \frac{\omega}{c}-i k \varepsilon_{1}^{(\lambda)}(k)-i k_{2} \varepsilon_{2}^{(\lambda)}(k)-i k_{3} \varepsilon_{3}^{(\lambda)}(k)=0
\end{aligned}
$$




\section{QuANTIZATION OF ELECTROMAGNETIC Field In INERTIAL Frame}

Second, in inertial frame, the quantization of electromagnetic field is

$$
\begin{aligned}
& E_{x}=-\frac{\partial \phi}{\partial x}-\frac{1}{c} \frac{\partial A_{x}}{\partial t} \\
& =\int \frac{d^{3} k}{(2 \pi)^{3} 2 k_{0}} \sum_{\lambda=0}^{3} \bar{\varepsilon}_{1}^{(\lambda)}(k)\left[a^{(\lambda)}(k) \exp (-k \cdot f+i \omega t)-a^{(\lambda)+}(k) \exp (k \cdot f-i \omega t)\right] \\
& \bar{\varepsilon}_{1}^{(\lambda)}(k)=i k \varepsilon_{0}^{(\lambda)}(k)-\frac{i}{c} \omega \varepsilon_{1}^{(\lambda)}(k)
\end{aligned}
$$

Hence, the quantization of magnetic field is in $\mathrm{X}$-axis

$$
\begin{aligned}
& B_{x}=\frac{\partial A_{z}}{\partial y}-\frac{\partial A_{y}}{\partial z} \\
&= \int \frac{d^{3} k}{(2 \pi)^{3} 2 k_{0}} \sum_{\lambda=0}^{3} \bar{\varepsilon}_{1}^{(\lambda)}(k)\left[a^{(\lambda)}(k) \exp (-k \cdot \rho+i \omega t)-a^{(\lambda)+}(k) \exp (k \cdot \rho-i \omega t)\right] \\
& \bar{\varepsilon}_{1}^{(\lambda)}(k)=-i k_{2} \varepsilon_{3}^{(\lambda)}(k)+i k_{3} \varepsilon_{2}^{(\lambda)}(k)
\end{aligned}
$$

So, the quantization of electric field is in Y-axis

$$
\begin{aligned}
& E_{y}=-\frac{\partial \phi}{\partial y}-\frac{1}{c} \frac{\partial A_{y}}{\partial t} \\
& =\int \frac{d^{3} k}{(2 \pi)^{3} 2 k_{0}} \sum_{\lambda=0}^{3} \bar{\varepsilon}_{2}^{(\lambda)}(k)\left[a^{(\lambda)}(k) \exp (-j \cdot f+i \omega t)-a^{(\lambda)+}(k) \exp (k \cdot f-i \omega t)\right] \\
& \bar{\varepsilon}_{2}^{(\lambda)}(k)=i k_{2} \varepsilon_{0}^{(\lambda)}(k)-\frac{i}{c} \omega \varepsilon_{2}^{(\lambda)}(k)
\end{aligned}
$$

Therefore, the quantization of magnetic field is in Y-axis

$$
\begin{gathered}
B_{y}=\frac{\partial A_{x}}{\partial z}-\frac{\partial A_{z}}{\partial x} \\
=\int \frac{d^{3} k}{(2 \pi)^{3} 2 k_{0}} \sum_{\lambda=0}^{3} \bar{\varepsilon}_{2}^{\prime(\lambda)}(k)\left[a^{(\lambda)}(k) \exp (-k \cdot f+i \omega t)-a^{(\lambda)+}(k) \exp (k \cdot f-i \omega t)\right] \\
\bar{\varepsilon}_{2}^{(\lambda)}(k)=-i k_{3} \varepsilon_{1}^{(\lambda)}(k)+i k \varepsilon_{3}^{(\lambda)}(k)
\end{gathered}
$$

Hence, the quantization of electric field is in Z-axis

$$
\begin{gathered}
E_{z}=-\frac{\partial \phi}{\partial z}-\frac{1}{c} \frac{\partial A_{z}}{\partial t} \\
=\int \frac{d^{3} k}{(2 \pi)^{3} 2 k_{0}} \sum_{\lambda=0}^{3} \bar{\varepsilon}_{3}^{(\lambda)}(k)\left[a^{(\lambda)}(k) \exp (-k \cdot f+i \omega t)-a^{(\lambda)+}(k) \exp (k \cdot f-i \omega t)\right] \\
\bar{\varepsilon}_{3}^{(\lambda)}(k)=i k_{3} \varepsilon_{0}^{(\lambda)}(k)-\frac{i}{c} \omega \varepsilon_{3}^{(\lambda)}(k)
\end{gathered}
$$


Therefore, the quantization of magnetic field is in Z-axis

$$
\begin{aligned}
& B_{z}=\frac{\partial A_{y}}{\partial x}-\frac{\partial A_{x}}{\partial y} \\
& =\int \frac{d^{3} k}{(2 \pi)^{3} 2 k_{0}} \sum_{\lambda=0}^{3} \bar{\varepsilon}_{3}^{(\lambda)}(k)\left[a^{(\lambda)}(k) \exp (-k \cdot \ell+i \omega t)-a^{(\lambda)+}(k) \exp (k \cdot \ell-i \omega t)\right] \\
& \bar{\varepsilon}_{3}^{(\lambda)}(k)=-i k \varepsilon_{2}^{(\lambda)}(k)+i k_{2} \varepsilon_{1}^{(\lambda)}(k)
\end{aligned}
$$

\section{Quantization OF EleCtromagnetic Field In RINDLER SPACE-Time}

Third, in the accelerated frame, the quantization of electromagnetic field is[1]

$$
\begin{aligned}
& E_{\xi^{1}}=-\frac{1}{\left(1+\frac{a_{0} \xi^{1}}{c^{2}}\right)} \frac{\partial}{\partial \xi^{1}}\left\{\left(1+\frac{a_{0} \xi^{1}}{c^{2}}\right)^{2} \phi_{\xi}\right\}-\frac{1}{\left(1+\frac{a_{0} \xi^{1}}{c^{2}}\right)} \frac{1}{c} \frac{\partial A_{\xi^{1}}}{\partial \xi^{0}}=E_{x} \\
& =\int \frac{d^{3} k}{(2 \pi)^{3} 2 k_{0}} \sum_{\lambda=0}^{3} \bar{\varepsilon}_{\xi^{1}}^{(\lambda)}(k)\left[a^{(\lambda)}(k) \exp (-k \cdot f+i \omega t)-a^{(\lambda)+}(k) \exp (k \cdot \ell-i \omega t)\right] \\
& \bar{\varepsilon}_{\xi^{\prime}}^{(\lambda)}(k)=\bar{\varepsilon}_{1}^{(\lambda)}(k)=i k \varepsilon_{0}^{(\lambda)}(k)-\frac{i}{c} \omega \varepsilon_{1}^{(\lambda)}(k)
\end{aligned}
$$

Hence, the quantization of magnetic field is in X-axis in the accelerated frame,

$$
\begin{aligned}
& B_{\xi^{1}}=\frac{\partial A_{\xi^{3}}}{\partial \xi^{2}}-\frac{\partial A_{\xi^{2}}}{\partial \xi^{3}}=B_{x} \\
&= \int \frac{d^{3} k}{(2 \pi)^{3} 2 k_{0}} \sum_{\lambda=0}^{3} \bar{\varepsilon}_{\xi^{\prime}}^{(\lambda)}(k)\left[a^{(\lambda)}(k) \exp (-k \cdot \ell+i \omega t)-a^{(\lambda)+}(k) \exp (k \cdot \ell-i \omega t)\right] \\
& \bar{\varepsilon}_{\xi^{\prime}}^{(\lambda)}(k)=\bar{\varepsilon}_{1}^{(\lambda)}(k)=-i k_{2} \varepsilon_{3}^{(\lambda)}(k)+i k_{3} \varepsilon_{2}^{(\lambda)}(k)
\end{aligned}
$$

So, the quantization of electric field is in Y-axis in the accelerated frame.

$$
\begin{aligned}
& E_{\xi^{2}}=-\frac{1}{\left(1+\frac{a_{0} \xi^{1}}{c^{2}}\right)} \frac{\partial}{\partial \xi^{2}}\left\{\left(1+\frac{a_{0} \xi^{1}}{c^{2}}\right)^{2} \phi_{\xi}\right\}-\frac{1}{\left(1+\frac{a_{0} \xi^{1}}{c^{2}}\right)} \frac{1}{c} \frac{\partial A_{\xi^{1}}}{\partial \xi^{0}} \\
= & -\left(1+\frac{a_{0} \xi^{1}}{c^{2}}\right) \frac{\partial \phi_{\xi}}{\partial \xi^{2}}-\frac{1}{\left(1+\frac{a_{0} \xi^{1}}{c^{2}}\right)} \frac{1}{c} \frac{\partial A_{\xi^{2}}}{\partial \xi^{0}}=E_{y} \cosh \left(\frac{a_{0} \xi^{0}}{c}\right)-B_{z} \sinh \left(\frac{a_{0} \xi^{0}}{c}\right) \\
= & \int \frac{d^{3} k}{(2 \pi)^{3} 2 k_{0}} \sum_{\lambda=0}^{3} \bar{\varepsilon}_{\xi^{2}}^{(\lambda)}(k)\left[a^{(\lambda)}(k) \exp \left(-\dot{\rho} \cdot \frac{\rho}{x}+\dot{\omega} t\right)-a^{(\lambda)+}(k) \exp (k \cdot \dot{\rho}-\dot{\omega} t)\right] \\
& \bar{\varepsilon}_{\xi^{2}}^{(\lambda)}(k)=\cosh \left(\frac{a_{0} \xi^{0}}{c}\right) \bar{\varepsilon}_{2}^{(\lambda)}(k)-\sinh \left(\frac{a_{0} \xi^{0}}{c}\right) \bar{\varepsilon}_{3}^{(\lambda)}(k) \\
= & \left.\cosh \left(\frac{a_{0} \xi^{0}}{c}\right)\left(i k_{2} \varepsilon_{0}^{(\lambda)}(k)-\frac{i}{c} \omega \varepsilon_{2}^{(\lambda)}(k)\right)-\sinh \frac{a_{0} \xi^{0}}{c}\right)\left(-i k \varepsilon_{2}^{(\lambda)}(k)+i k_{2} \varepsilon_{1}^{(\lambda)}(k)\right)
\end{aligned}
$$


Therefore, the quantization of magnetic field is in Y-axis in the accelerated frame,

$$
\begin{aligned}
& B_{\xi^{2}}=\frac{\partial A_{\xi^{1}}}{\partial \xi^{3}}-\frac{\partial A_{\xi^{3}}}{\partial \xi^{1}}=B_{y} \cosh \left(\frac{a_{0} \xi^{0}}{c}\right)+E_{z} \sinh \left(\frac{a_{0} \xi^{0}}{c}\right) \\
& =\int \frac{d^{3} k}{(2 \pi)^{3} 2 k_{0}} \sum_{\lambda=0}^{3} \bar{\varepsilon}_{\xi^{2}}^{(\lambda)}(k)\left[a^{(\lambda)}(k) \exp (-k \cdot \rho+i \omega t)-a^{(\lambda)+}(k) \exp (k \cdot \rho-i \omega t)\right] \\
& \bar{\varepsilon}_{\xi^{2}}^{(\lambda)}(k)=\cosh \left(\frac{a_{0} \xi^{0}}{c}\right) \bar{\varepsilon}_{2}^{(\lambda)}(k)+\sinh \left(\frac{a_{0} \xi^{0}}{c}\right) \bar{\varepsilon}_{3}^{(\lambda)}(k) \\
& \left.=\cosh \left(\frac{a_{0} \xi^{0}}{c}\right)\left(-i k_{3} \varepsilon_{1}^{(\lambda)}(k)+i k \varepsilon_{3}^{(\lambda)}(k)\right)+\sinh \frac{a_{0} \xi^{0}}{c}\right)\left(i k_{3} \varepsilon_{0}^{(\lambda)}(k)-\frac{i}{c} \omega \varepsilon_{3}^{(\lambda)}(k)\right)
\end{aligned}
$$

Hence, the quantization of electric field is in Z-axis in the accelerated frame,

$$
\begin{aligned}
& E_{\xi^{3}}=-\frac{1}{\left(1+\frac{a_{0} \xi^{1}}{c^{2}}\right)} \frac{\partial}{\partial \xi^{3}}\left\{\left(1+\frac{a_{0} \xi^{1}}{c^{2}}\right)^{2} \phi_{\xi}\right\}-\frac{1}{\left(1+\frac{a_{0} \xi^{1}}{c^{2}}\right)} \frac{1}{c} \frac{\partial A_{\xi^{1}}}{\partial \xi^{0}} \\
& =-\left(1+\frac{a_{0} \xi^{1}}{c^{2}}\right) \frac{\partial \phi_{\xi}}{\partial \xi^{3}}-\frac{1}{\left(1+\frac{a_{0} \xi^{1}}{c^{2}}\right)} \frac{1}{c} \frac{\partial A_{\xi^{3}}}{\partial \xi^{0}}=E_{z} \cosh \left(\frac{a_{0} \xi^{0}}{c}\right)+B_{y} \sinh \left(\frac{a_{0} \xi^{0}}{c}\right) \\
& =\int \frac{d^{3} k}{(2 \pi)^{3} 2 k_{0}} \sum_{\lambda=0}^{3} \bar{\varepsilon}_{\xi^{3}}^{(\lambda)}(k)\left[a^{(\lambda)}(k) \exp (-\dot{k} \cdot f+i \omega t)-a^{(\lambda)+}(k) \exp (j \cdot \rho-\dot{k} t)\right] \\
& \bar{\varepsilon}_{\xi^{3}}^{(\lambda)}(k)=\cosh \left(\frac{a_{0} \xi^{0}}{c}\right) \bar{\varepsilon}_{3}^{(\lambda)}(k)+\sinh \left(\frac{a_{0} \xi^{0}}{c}\right) \bar{\varepsilon}_{2}^{(\lambda)}(k) \\
& =\cosh \left(\frac{a_{0} \xi^{0}}{c}\right)\left(i k_{3} \varepsilon_{0}^{(\lambda)}(k)-\frac{i}{c} \omega \varepsilon_{3}^{(\lambda)}(k)\right)+\sinh \left(\frac{a_{0} \xi^{0}}{c}\right)\left(i k_{3} \varepsilon_{1}^{(\lambda)}(k)+i k \varepsilon_{3}^{(\lambda)}(k)\right)
\end{aligned}
$$

Therefore, the quantization of magnetic field is in Z-axis in the accelerated frame,

$$
\begin{aligned}
& B_{\xi^{3}}=\frac{\partial A_{\xi^{2}}}{\partial \xi^{1}}-\frac{\partial A_{\xi^{1}}}{\partial \xi^{2}}=B_{z} \cosh \left(\frac{a_{0} \xi^{0}}{c}\right)-E_{y} \sinh \left(\frac{a_{0} \xi^{0}}{c}\right) \\
& =\int \frac{d^{3} k}{(2 \pi)^{3} 2 k_{0}} \sum_{\lambda=0}^{3} \bar{\varepsilon}_{\xi^{3}}^{(\lambda)}(k)\left[a^{(\lambda)}(k) \exp (-k \cdot f+i \omega t)-a^{(\lambda)+}(k) \exp (k \cdot f-i \omega t)\right] \\
& \quad \vec{\varepsilon}_{\xi^{3}}^{(\lambda)}(k)=\cosh \left(\frac{a_{0} \xi^{0}}{c}\right) \vec{\varepsilon}_{3}^{(\lambda)}(k)-\sinh \left(\frac{a_{0} \xi^{0}}{c}\right) \bar{\varepsilon}_{2}^{(\lambda)}(k) \\
& =\cosh \left(\frac{a_{0} \xi^{0}}{c}\right)\left(-i k \varepsilon_{2}^{(\lambda)}(k)+i k_{2} \varepsilon_{1}^{(\lambda)}(k)\right)-\sinh \left(\frac{a_{0} \xi^{0}}{c}\right)\left(i k_{2} \varepsilon_{0}^{(\lambda)}(k)-\frac{i}{c} \omega \varepsilon_{2}^{(\lambda)}(k)\right)
\end{aligned}
$$

\section{CONCLuSiON}

Therefore, according to [1], likely the electromagnetic field, the potential did quantizated in inertial frame, the electromagnetic field, the potential can quantizate by the transformation of electromagnetic field or the transformation of the potential in the accelerated frame 


\section{REFERENCES}

[1] S.Yi, "Electromagnetic Field Equation and Lorentz Gauge in Rindler space-time", The African review of physics, 11,33(2016)-INSPIRE-HEP

[2] S.Yi, "Electromagnetic Wave Function and Equation, Lorentz Force in Rindler space-time", International Journal of Advanced Research in Physical Science,5,9(2018)

[3] C.Itzykson \& J.B.Zuber, Quantum Field Theory, McGraw-Hill,(1980)

[4] S.S. Schweber, An Introduction to Relativistic Quantum Field Theory, Haper and Row,(1962)

[5] G.Kallen,Quantum Electrodynamics, Springer-Verlag,(1972)

[6] T.D.Lee, Particle Physics and Introduction to Field Theory, Ch.2 Harwood Academic Publisher,(1981)

[7] S.Weinberg,Gravitation and Cosmology(John wiley \& Sons,Inc,1972)

[8] W.Rindler, Am.J.Phys.34.1174(1966)

[9] P.Bergman,Introduction to the Theory of Relativity(Dover Pub. Co.,Inc., New York, 1976), Chapter V

[10] C.Misner, K,Thorne and J. Wheeler, Gravitation(W.H.Freedman \& Co.,1973)

[11] S.Hawking and G. Ellis, The Large Scale Structure of Space-Time(Cam-bridge University Press, 1973)

[12] R.Adler,M.Bazin and M.Schiffer,Introduction to General Relativity(McGraw-Hill,Inc.,1965)

[13] W.Rindler, Special Relativity(2nd ed., Oliver and Boyd, Edinburg,1966)

[14] Massimo Pauri, Michele Vallisner, "Marzke-Wheeler coordinates for accelerated observers in special relativity":Arxiv:gr-qc/0006095(2000)

Citation: Sangwha-Yi, (2019). Quantization of Electromagnetic Field and Potential in Rindler Space-Time. International Journal of Advanced Research in Physical Science (IJARPS) 6(5), pp.14-19, 2019.

Copyright: (C) 2019 Authors, this is an open-access article distributed under the terms of the Creative Commons Attribution License, which permits unrestricted use, distribution, and reproduction in any medium, provided the original author and source are credited. 